\title{
Wavelet and FFT Based Image Denoising Using Non-Linear Filters
}

\author{
S. Gopinathan*, R. Kokila*, P. Thangavel** \\ Department of Computer Science, University of Madras, Chepauk, Chennai - 600005, India
}

\begin{tabular}{l}
\hline \hline Article Info \\
\hline Article history: \\
Received Feb 13, 2015 \\
Revised Jun 28, 2015 \\
Accepted Jul 14, 2015 \\
\hline Keyword: \\
Cubic spline interpolation \\
Fast Fourier transform \\
Lagrange interpolation \\
Piecewise linear filter \\
Soft thresholding
\end{tabular}

\begin{abstract}
We propose a stationary and discrete wavelet based image denoising scheme and an FFT based image denoising scheme to remove Gaussian noise. In the first approach, high subbands are added with each other and then soft thresholding is performed. The sum of low subbands is filtered with either piecewise linear (PWL) or Lagrange or spline interpolated PWL filter. In the second approach, FFT is employed on the noisy image and then low frequency and high frequency coefficients are separated with a specified cutoff frequency. Then the inverse of low frequency components is filtered with one of the PWL filters and the inverse of high frequency components is filtered with soft thresholding. The experimental results are compared with Liu and Liu's tensor-based diffusion model (TDM) approach.
\end{abstract}

Copyright () 2015 Institute of Advanced Engineering and Science. All rights reserved.

\section{Corresponding Author:}

P. Thangavel,

Head of the department, Department of Computer Science, University of Madras, Chepauk, Chennai 600005, India.

Email: thangavelp@yahoo.com

\section{INTRODUCTION}

Images acquired by image sensors may be corrupted by noise due to noisy sensors, faulty CCD elements and dust on the lens [1-2]. Noise could degrade the quality of the image and also might result in loss of important data. Furthermore, noise can be introduced by transmission errors and also by different compression methods. Therefore, denoising is often vital and first step to be performed before images are being analyzed. It has remained a fundamental problem for researchers because noise removal introduces artifacts and causes blurring of the image.

Many image denoising methods that have been proposed and studied. They can be classified into two groups [3]: the spatial domain filtering and the transform domain filtering. In spatial domain filtering, spatial filters are applied to the image data in order to remove the noise. These filters remove noise to a reasonable extent but at the cost of blurring images which in turn makes the edges in picture invisible. Various non-linear filters such as weighted median [4], rank conditioned rank section [5] and relaxed median [6] were introduced to overcome this drawback. Russo [7] proposed an image enhancement system using piecewise linear filter for directional smoothing. Thangavel and Gopinathan [8] proposed an image enhancement scheme using wavelet transform and smooth approximation of a piecewise linear (PWL) filter. In frequency domain [9] the removal of noise is achieved by designing a frequency domain filter and adapting a cutoff frequency when the noise components are decorrelated from the useful signal domain. Independent component analysis method was implemented in [10] for denoising of non Gaussian data.

An image resolution enhancement technique using discrete wavelet transform and stationary wavelet transform was proposed by Demirel et al. [11]. Chouhan et al. [12] proposed wavelet based blind watermarking in order to overcome the problem of false matching of fingerprint. Jaiswal et al. [13] denoised the image by using filtering method and then applied wavelet based technique using threshold. Chan and Ma [14] proposed a box constrained multiplicative iterative algorithm for box constrained image resolution. A 
local and contextual controlled (LCC) fourth order Partial differential equation method for the noise removal was presented by Zeng et al. [15]. Lang et al. [16] presented a new nonlinear noise reduction method that uses the discrete wavelet transform. Clyde et al. [17] discussed about bayesian methods for multiple shrinkage estimation in wavelets. Dabov et al. [18] proposed an image denoising strategy based on an enhanced sparse representation in transform domain. Yang et al. [19] proposed an image enhancement approach using wavelet transform and Haar transform for medical image denoising. Different thresholding procedures were analysed by Lázaro et al. [20] using the discrete wavelet transform and decomposition level dependent threshold. Donoho [21] proposed a soft thresholding method for denoising an image. Khare et al. [22] proposed a method for denoising medical images using soft thresholding in wavelet domain on multiple levels. Luisier et al. [23] introduced a new approach based on interscale orthonormal wavelet thresholding.

Lu et al. [24] investigated an adaptive scheme based on the nonlocal means (NL-means) algorithm for medical image denoising. Shang and Huang [25] proposed a method for denoising using extended nonnegative sparse coding neural network shrinkage algorithm. Adler et al. [26] proposed a new approach that optimizes the shape of the shrinkage functions and maximizes denoising performance by emphasizing the contribution of sparse overcomplete representations. Liu and Liu [1] have proposed image denoising based on diffusion tensors. Jianwei [28] proposed an algorithm based on pixel processing for the noise removal of color images. Jain and Tyagi [29] proposed locally adaptive patch based (LAPD) method which reduces the noise while preserving the releavant features of the image. Inspite of these methods, the problem attracts many researchers because of its practical importance and even a small improvement in results would yield a high order of significance. So we have considered the problem in general not specific to any particular application.

In this paper we propose two approaches, to remove noise from an image. The first approach uses stationary wavelet transform (SWT) and discrete wavelet transform (DWT) where as the second approach uses fast Fourier transform (FFT). The proposed schemes use PWL or its smooth approximation for smoothing slowly varying components and soft thresholding for sharpening edge related pixels. We discuss about piecewise linear filter and its smooth approximations in Section 2. The soft thresholding technique is presented in Section 3. We describe image denoising approach using SWT and DWT in Section 4. FFT based image denoising scheme is presented in Section 5. Experimental results are discussed in Section 6. Finally the conclusions are drawn in Section 7.

\section{PIECEWISE LINEAR FILTER AND ITS SMOOTH APPROXIMATIONS}

In this section, we review PWL filter proposed by Russo [7] for smoothing non edge pixels. The processing deals with four different subset of pixels $W_{1}, W_{2}, W_{3}$ and $W_{4}$.

$$
\begin{aligned}
& W_{1}=\left\{x_{i-2, j}, x_{i-1, j}, x_{i+1, j}, x_{i+2, j}\right\} \\
& W_{1}=\left\{x_{i, j-2}, x_{i, j-1}, x_{i, j+1}, x_{i, j+2}\right\} \\
& W_{1}=\left\{x_{i-2, j-2}, x_{i-1, j-1}, x_{i+1, j+1}, x_{i+2, j+2}\right\} \\
& W_{1}=\left\{x_{i+2, j-2}, x_{i+1, j-1}, x_{i-1, j+1}, x_{i-2, j+2}\right\}
\end{aligned}
$$

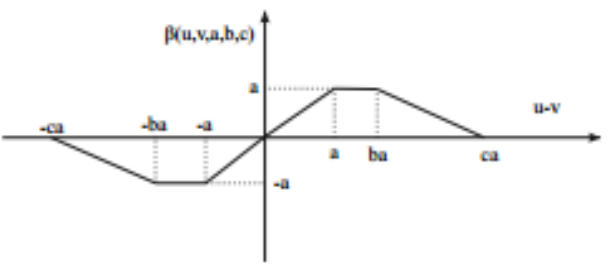

(a)

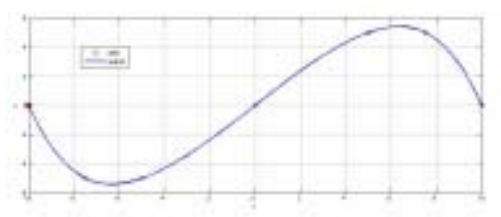

(c)

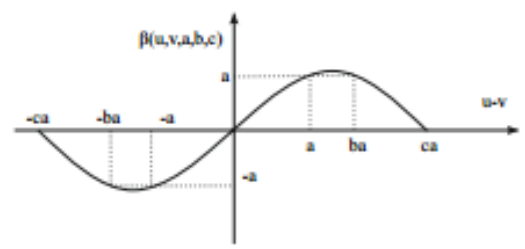

(b)

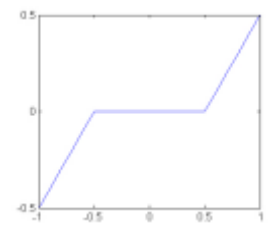

(d)

Figure 1. (a) Piecewise linear (PWL) filter; (b) Lagrange interpolated PWL filter; (c) Spline interpolated PWL filter; (d) Soft threshold function for $\mathrm{T}=\frac{1}{2}$ 
The output $\mathcal{Y}_{i, j}$ of this filter is given by the relation

$$
y_{i, j}=x_{i, j}+\frac{1}{4} \sum_{x_{m, c} \in W_{p}} \beta\left(x_{m, n}, x_{i, j}, a, b, c\right)
$$

where $\beta\left(x_{m, n}, x_{i, j}, a, b, c\right)$ is the function of the three parameter seven segment PWL function shown in Figure $1(\mathrm{a})$ and the set $W_{p}$ is selected such that the following quantity is minimized.

$$
\min _{k=1,2,3,4}\left|\sum_{x_{m, n} \in W_{k}} \beta\left(x_{m, n}, x_{i, j}, a, b, c\right)\right|
$$

Based on the filtering scheme defined by (1) and (2) only one pixel subset is selected. The filtering operation is performed by restoring to the minimum operator. To further reduce the noise, another pixel subset is selected, by restoring to the maximum operator which is defined by (3) and (4).

$$
\begin{aligned}
& y_{i, j}=x_{i, j}+\frac{1}{4} \sum_{x_{m, n} \in W_{q}} \beta\left(x_{m, n}, x_{i, j}, a, b, c\right) \\
& \max _{k=1,2,3,4}\left|\sum_{x_{m, n} \in W_{k}} \beta\left(x_{m, n}, x_{i, j}, a, b, c\right)\right|
\end{aligned}
$$

Russo [7] designed the PWL filter based on the range of luminance using the parameters $a$; $b$ and $c$. The lengths of all segments of the PWL function are controlled by parameter $a$. For small luminance differences $(|u-v| \leq a)$ which are interpreted as noise, the filter should perform strong smoothing. A gradual transition is required when the luminance differences are medium $(a \leq|u-v| \leq b a)$ and mediumlarge ( $b a \leq|u-v| \leq c a)$. Such transition can be achieved by using the trapezoid-shaped function so that the filter can perform weak smoothing. Smoothing is not necessary for large luminance $(|u-v|>c a)$. The range for the parameters $b$ and $c$ are $1 \leq b \leq 2$ and $2 \leq c \leq 5$ respectively. The parameter a depends on the noise variance. Here we have used $b=1.5$ and $c=5$.

\subsection{Lagrange Interpolation}

Let $x_{0}, x_{1} ; x_{2}, \ldots, x n$ be $n+1$ distinct points on the real axis and let $\mathrm{f}(x)$ be a real valued function defined on some interval $I=[a, b]$ containing these points. We can construct a polynomial $p(x)$ of degree $\leq n$ which interpolates $f(x)$ at the $n+1$ distinct points $x_{0}, x_{1}, x_{2}, \ldots, x_{n}$ [27] as follows:

$$
\begin{aligned}
& p(x)=a_{0} l_{0}(x)+a_{1} l_{1}(x)+\cdots+a_{n} l_{n}(x) \\
& l_{k}(x)=\prod_{\substack{i=0 \\
i \neq k}}^{n} \frac{x-x_{i}}{x_{k}-x_{i}}
\end{aligned}
$$

Using (5) the PWL function is smoothed for the parameters $b=1.5$ and $c=5$ and is shown in Figure 1(b). The smoothed version of PWL function is used to smooth non edge pixels of the input image.

\subsection{Cubic Spline Interpolation}

We can also obtain the smooth approximation of piecewise linear function using cubic spline interpolation. For the above selected parameters the interpolated curve is shown in Figure 1(c).

\section{SOFT THRESHOLDING}

A soft threshold is a preprocessing tool that reduces the background in an image, so that the pixels with intensity values below the threshold value are reduced. During visualization, these thresholded pixels become more transparent. Soft threshold sets zero the elements whose absolute values are lower than threshold, then shrinks the other co-efficients towards zero.

The soft threshold was proposed by Donoho [21]. Later Yang et al. [19] used the following threshold value to calculate the soft threshold: 
$\operatorname{Treshold}(T)=\sigma \sqrt{2 \log (W \cdot H)}$

$\sigma=\sqrt{\frac{1}{W . H} \sum_{i=1}^{W} \sum_{i=1}^{H}\left(S_{i, j}-\bar{S}\right)^{2}}$

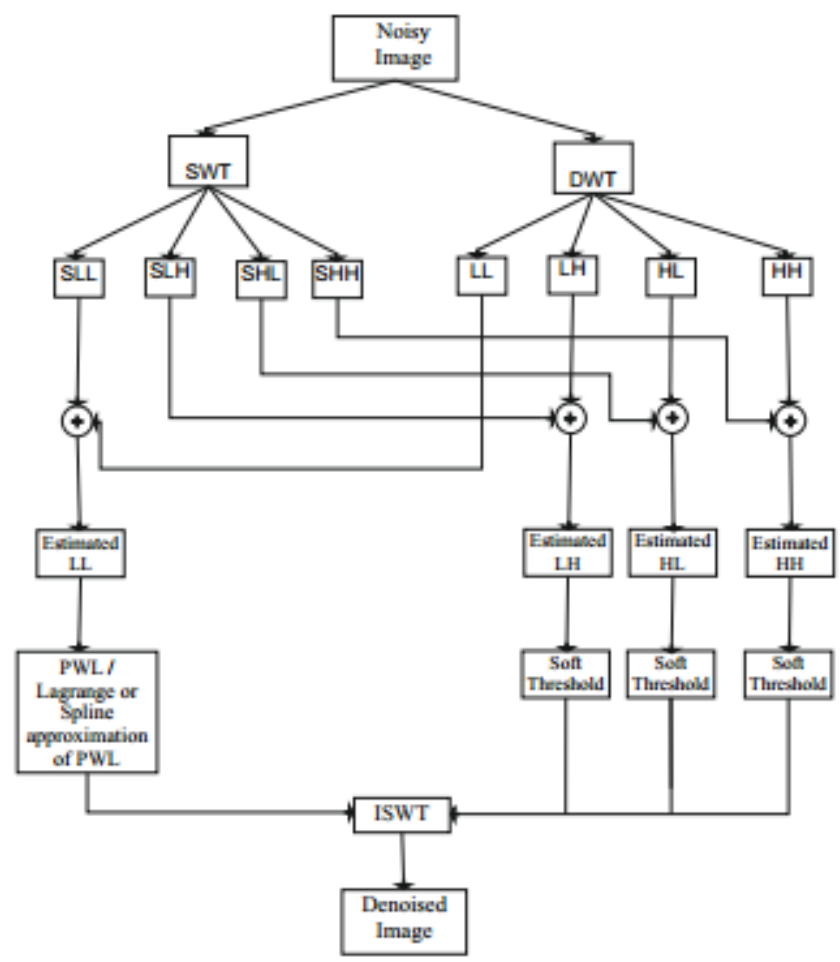

Figure 2. Block diagram of image denoising using SWT and DWT scheme.

where $\mathrm{W}$ and $\mathrm{H}$ are the width and height of $S^{\text {th }}$ subband and $\bar{S}$ is the mean of $S^{\text {th }}$ subband. We have used the above formula to compute soft threshold in our experiments. Soft threshold is used to remove the noise using soft shrinkage rule which is given by

$$
\rho T(x)=\left\{\begin{array}{cc}
x+T, & \text { if } x \leq-\mathrm{T} \\
0, & \text { if }|x|<\mathrm{T} \\
x-T & \text { if } x>\mathrm{T}
\end{array}\right.
$$

For example, the graph for threshold function with $\mathrm{T}=\frac{1}{2}$ is illustrated in Figure $1(\mathrm{~d})$.

\section{IMAGE DENOISING USING SWT AND DWT}

We consider images added with Gaussian noise for different values of standard deviation $(\sigma)$. Discrete wavelet transform is applied to decompose the noisy image into different subband images. LH (Low-High), HL (High-Low), HH (High-High) are the three high subbands, each of which contains the high frequency components of the input image. LL (Low-Low) subband contains the low frequency components of the input image. Bicubic interpolation with interpolation factor of 2 is applied to all frequency subband images of DWT. Similarly SWT is applied to the noisy image, which decomposes the image into four different subbands namely SLL, SLH, SHL and SHH. In this decomposition all the subbands will be of same size. Now we add the corresponding subbands of SWT and DWT with each other. 
For the estimated low frequency subband the PWL or Lagrange or cubic spline interpolated PWL filter is applied to improve the smoothness and remove noise. Soft thresholding is used to enhance the sharpness of the remaining three high frequency subband image. Inverse SWT is applied for the resulting frequency subbands to retrieve the denoised image. The block diagram of the scheme is shown in Figure 2.

\section{FFT BASED IMAGE DENOISING}

First the fast Fourier transform is applied to the noise added image to transform the image from the spatial domain to frequency domain. A lowpass filter and a highpass filter are designed. Then the transformed image is filtered with lowpass filter and highpass filter. Now the inverse fast Fourier transform is employed to the lowpass filtered image and highpass filtered image. Soft thresholding is applied to the inverse of highpass image in order to enhance the sharpness of the image. The PWL filter or Lagrange or spline interpolated PWL filter is applied to the inverse of lowpass image, to enhance the smoothness of the image. Then the resulting two images are combined to retrieve the denoised image. The block diagram of FFT based image denoising scheme is shown in Figure 3.

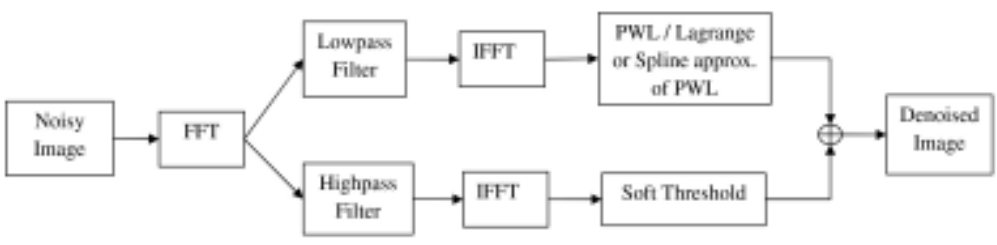

Figure 3. Block diagram of FFT based image denoising scheme

\subsection{Lowpass Filter}

The transformed image in the frequency domain near the center will have low frequency components. The lowpass filter [2] can be constructed using the following formula:

$$
H_{I L P}(\mu, \gamma)= \begin{cases}1, & \text { if } D(\mu, \gamma) \leq f_{c} \\ 0, & \text { if } D(\mu, \gamma)>f_{c}\end{cases}
$$

where $\mathrm{D}(\mu, \gamma)$ is the distance between the center of the frequency rectangle and a point $(\mu, \gamma)$ in the frequency domain:

$$
D(\mu, \gamma)=\sqrt{(\mu-W / 2)^{2}+(\gamma-H / 2)^{2}}
$$

here $W$ and $H$ are the width and height of the image respectively. We have set the cutoff frequency, $f_{c}=200$, as we have used images of size 512 x 512 .

\subsection{Highpass Filter}

In the transformed image, the region near the edge will have high frequency components than the central region. Figure 4 shows the pictorial representation of these filters. The highpass filter can be constructed by using their corresponding lowpass filter:

$$
H_{I L P}(\mu, \gamma)=1-H_{I L P}(\mu, \gamma)
$$

\section{EXPERIMENTAL RESULTS}

The simplest and most widely used performance measures are peak signal to noise ratio ( $P S N R$ ) and mean squared error (MSE). Let $f(x, y)$ and $\hat{f}(x, y)$ are the reference and test images respectively. Let $e(x, y)$ be the error signal between $f(x, y)$ and $\hat{f}(x, y)$. It is computed pixel-by-pixel by adding up the squared ifferences of all the pixels and dividing by the total pixel count. If $\mathrm{M} \mathrm{x} \mathrm{N}$ is the size of the image, then 
$M S E=\frac{1}{M N} \sum_{x=1}^{M} \sum_{y=1}^{N}(e(x, y))^{2}=\frac{1}{M N} \sum_{x=1}^{M} \sum_{y=1}^{N}(f(x, y)-\hat{f}(x, y))^{2}$

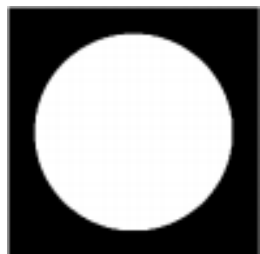

(a)

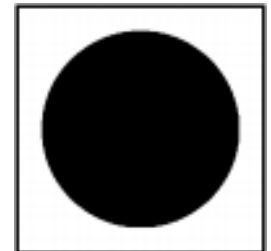

(b)

Figure 4. (a) Lowpass filter; (b) Highpass filter

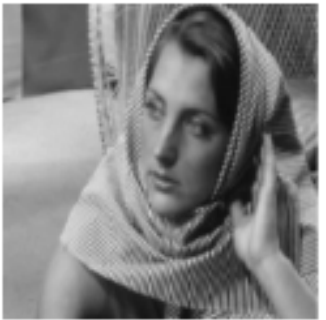

(a)

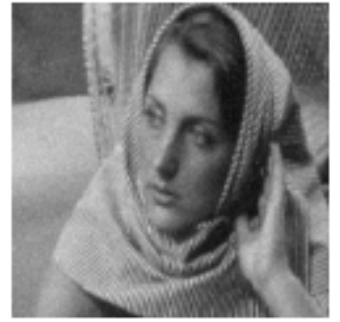

(e)

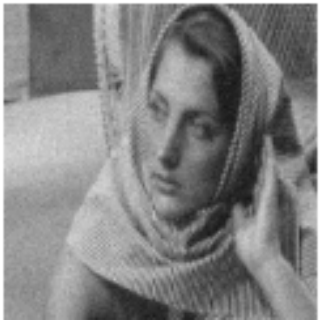

(b)

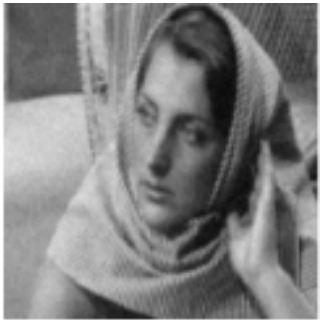

(f)

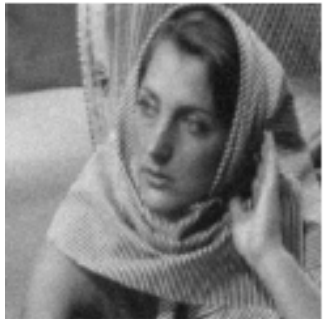

(c)

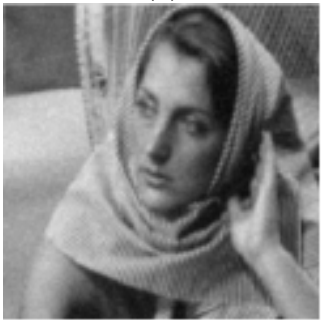

(g)

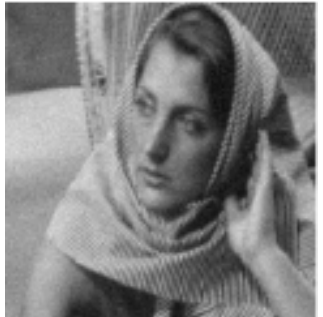

(d)

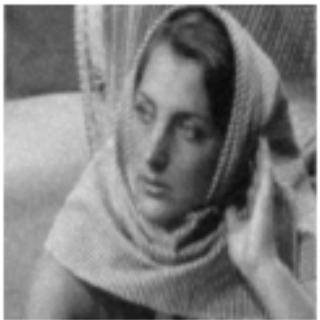

(h)

Figure 5. Image denoising scheme with = 40 for Barbara image: (a) Original Image; (b) Noisy Image; Enhanced images using FFT based scheme with (c) Lagrange interpolated PWL filter, (d) spline interpolated PWL filter, (e) PWL filter; Enhanced images using SWT-DWT based scheme with (f) Lagrange interpolated PWL filter, (g) spline interpolated PWL filter, (h) PWL filter

The P S N R quality assessment metric is defined as follows:

$$
P S N R=10 \log _{10} \frac{M A X_{1}^{2}}{M S E}
$$

here, $M A X_{I}$ is the maximum possible pixel value of the image. The higher the $P S N R$, the closer the test image is to the original.

To evaluate the effectiveness of the proposed image denoising approaches, we used 12 grayscale images. All the test images are of size 512 x 512. Gaussian noise with standard deviation $(\sigma)$ is added to the input image. First, SWT and DWT based image denoising method is applied to the noisy image. Simultaneously FFT based image denoising scheme is also applied to the noisy image. To measure the performance of each scheme, we use $P S N R$ metric between the denoised image and original image. Figure 5 displays the results of FFT and SWT-DWT based image denoising schemes for Barbara image. The first row consists of original image, noisy image and results of FFT based image denoising scheme using Lagrange interpolation, spline interpolation, PWL filter respectively. The second row contains the results of SWT-DWT based image denoising scheme using Lagrange interpolation, spline interpolation and PWL filter respectively. 
The following grayscale images with Gaussian noise of specified $\sigma$ from $\{20,25,30,35,40,45\}$ are used in our experiments: Airplane $\left(I_{1}\right)$, Lena $\left(I_{2}\right)$, Goldhill $\left(I_{3}\right)$, Monarch $\left(I_{4}\right)$, Elaine $\left(I_{5}\right)$, Cameraman $\left(I_{6}\right)$, Barbara $\left(I_{7}\right)$, Pepper $\left(I_{8}\right)$, Couple $\left(I_{9}\right)$, Bridge $\left(I_{10}\right)$, Boat $\left(I_{11}\right)$ and 5.2.09 $\left(I_{12}\right)$. In the Table 1, we have shown P SNR values for the denoised images using FFT and SWT-DWT based schemes. Each column consists of two cells, one for FFT and other for SWT-DWT. From this table, we can observe that FFT method performs well with respect to $P S N R$ measures.

We have also compared SWT-DWT and FFT based image denoising scheme results of Lena and Boat images with Liu and Liu [1] scheme of tensor-based diffusion model (TDM) for different $\sigma$ values. Tables 2 and 3, show the P S N R values corresponds to TDM, SWT-DWT and FFT based schemes. From the results it can be observed that for different $\sigma$ values, FFT based scheme performs better than the other two schemes.

We also have used different cutoff frequencies such as 180; 190; 200; 210 and 220 for FFT based scheme with Lagrange interpolated PWL filter with Gaussian noise added images such as Airplane, Barbara, Elaine, Goldhill and Lena with the standard deviation of 30 and 50. The P SNR values for different cutoff frequencies are shown in Table 4 and Table 5 for $\sigma=30, \sigma=50$ respectively, and their corresponding pictorial representations are shown in Figure 6. From the pictorial representations, it can be observed that at cutoff frequency 200, the performance of the

Table 1. P SNR value for denoised images using FFT and SWT-DWT method. Each cell has P SNR value of FFT and SWT-DWT denoising scheme using Lagrange interpolation. Here $\sigma$ represents the standard deviation of the Gaussian noise added to Grayscale image.

\begin{tabular}{|c|c|c|c|c|c|c|c|c|c|c|c|c|}
\hline \multirow{3}{*}{$\begin{array}{c}\sigma \\
\text { Noisy }\end{array}$} & \multicolumn{2}{|c|}{20} & \multicolumn{2}{|c|}{25} & \multicolumn{2}{|c|}{30} & \multicolumn{2}{|c|}{35} & \multicolumn{2}{|c|}{40} & \multicolumn{2}{|c|}{45} \\
\hline & \multicolumn{2}{|c|}{26.88} & \multicolumn{2}{|c|}{24.94} & \multicolumn{2}{|c|}{23.36} & \multicolumn{2}{|c|}{22.02} & \multicolumn{2}{|c|}{20.86} & \multicolumn{2}{|c|}{19.84} \\
\hline & 33.08 & 25.09 & 31.86 & 25.18 & 30.99 & 24.92 & 30.36 & 25.70 & 29.33 & 26.80 & 26.88 & 26.78 \\
\hline $\mathrm{I}_{2}$ & 31.08 & 27.41 & 30.54 & 26.79 & 31.15 & 26.27 & 29.74 & 27.44 & 27.05 & 27.67 & 27.94 & 26.79 \\
\hline $\mathrm{I}_{3}$ & 34.04 & 27.93 & 30.39 & 27.88 & 29.80 & 28.28 & 29.95 & 29.57 & 27.27 & 27.91 & 28.18 & 28.89 \\
\hline $\mathrm{I}_{4}$ & 27.38 & 25.02 & 27.94 & 26.01 & 29.21 & 25.18 & 29.74 & 26.77 & 27.60 & 26.07 & 28.34 & 26.68 \\
\hline $\mathrm{I}_{5}$ & 29.70 & 27.08 & 29.41 & 26.35 & 27.83 & 25.05 & 27.51 & 25.49 & 27.51 & 26.24 & 27.67 & 27.27 \\
\hline $\mathrm{I}_{6}$ & 34.43 & 32.14 & 33.10 & 32.47 & 31.15 & 31.91 & 29.87 & 30.98 & 28.86 & 30.75 & 28.08 & 30.05 \\
\hline $\mathrm{I}_{7}$ & 30.76 & 24.83 & 30.36 & 25.08 & 29.02 & 25.17 & 29.18 & 25.00 & 28.64 & 25.13 & 28.07 & 25.41 \\
\hline $\mathrm{I}_{8}$ & 27.37 & 24.63 & 25.34 & 24.00 & 24.36 & 23.68 & 25.15 & 23.83 & 24.27 & 23.84 & 24.08 & 22.96 \\
\hline $\mathrm{I}_{9}$ & 31.13 & 27.66 & 30.00 & 27.55 & 29.15 & 27.37 & 28.68 & 27.09 & 27.84 & 26.92 & 27.38 & 26.55 \\
\hline $\mathrm{I}_{10}$ & 30.28 & 26.85 & 29.42 & 26.57 & 28.57 & 26.43 & 27.85 & 26.21 & 27.10 & 26.10 & 26.46 & 25.88 \\
\hline $\mathrm{I}_{11}$ & 34.43 & 28.52 & 33.02 & 28.67 & 31.45 & 27.43 & 29.44 & 26.83 & 29.82 & 26.76 & 28.81 & 26.82 \\
\hline $\mathrm{I}_{12}$ & 30.76 & 26.64 & 29.29 & 26.86 & 28.83 & 26.49 & 28.69 & 25.42 & 26.42 & 26.43 & 25.86 & 26.32 \\
\hline
\end{tabular}

Table 2. Comparison of P SNR values between TDM, SWT-DWT and FFT based methods for Boat image

\begin{tabular}{cccccccc}
\hline$\sigma$ & \multirow{2}{*}{ TDM } & PWL & $\begin{array}{c}\text { SWT-DWT } \\
\text { Spline }\end{array}$ & Lagrange & PWL & Spline & Lagrange \\
\hline 20 & 27.84 & 27.7507 & 27.7013 & 27.5782 & 31.3863 & 33.0488 & 34.0468 \\
30 & 26.16 & 27.5173 & 27.5255 & 27.3760 & 28.7439 & 30.3653 & 31.4244 \\
50 & 24.04 & 27.3142 & 27.5508 & 27.5042 & 26.7582 & 27.3454 & 28.0519 \\
\hline
\end{tabular}

Table 3. Comparison of $P S N R$ values between TDM, SWT-DWT and FFT based methods for Lena image

\begin{tabular}{|c|c|c|c|c|c|c|c|}
\hline \multirow{2}{*}{$\sigma$} & \multirow{2}{*}{ TDM } & \multicolumn{3}{|c|}{ SWT-DWT } & \multicolumn{3}{|c|}{ FFT } \\
\hline & & PWL & Spline & Lagrange & PWL & Spline & Lagrange \\
\hline 20 & 29.14 & 26.2591 & 27.4298 & 27.4133 & 26.7536 & 30.3549 & 31.4452 \\
\hline 30 & 27.29 & 26.6707 & 26.4133 & 26.4288 & 28.4684 & 29.3505 & 29.9958 \\
\hline 50 & 25.15 & 27.8283 & 28.4620 & 28.3426 & 26.4432 & 27.4142 & 27.8814 \\
\hline
\end{tabular}

Table 4. $P S N R$ values for different cutoff frequencies with FFT and Lagrange interpolated PWL filter based scheme with $\sigma=30$.

\begin{tabular}{cccccc}
\hline Cutoff & Airplane & Barbara & Elaine & GoldHill & Lena \\
\hline 180 & 23.9479 & 23.5926 & 23.9084 & 23.9758 & 23.9843 \\
190 & 26.5547 & 29.2991 & 27.9449 & 29.8900 & 28.6765 \\
200 & 30.9866 & 29.0209 & 27.8334 & 29.8032 & 31.1527 \\
210 & 26.5816 & 29.8457 & 27.2293 & 30.4966 & 28.4099 \\
220 & 25.9406 & 29.4629 & 28.0986 & 29.8876 & 27.7151 \\
\hline
\end{tabular}


Table 5. P SNR values for different cutoff frequencies with FFT and Lagrange interpolated PWL filter based

\begin{tabular}{cccccc}
\multicolumn{7}{c}{ scheme with $\sigma=50}$. \\
\hline Cutoff & Airplane & Barbara & Elaine & GoldHill & Lena \\
\hline 180 & 26.4769 & 27.5803 & 26.3209 & 28.6792 & 26.3410 \\
190 & 26.5011 & 27.6463 & 26.6938 & 28.6166 & 28.2330 \\
200 & 26.9071 & 27.3674 & 26.6707 & 27.7516 & 27.5388 \\
210 & 26.4511 & 27.1022 & 26.4052 & 27.9583 & 28.0599 \\
220 & 26.3734 & 27.4392 & 25.6567 & 27.8673 & 27.1952 \\
\hline
\end{tabular}

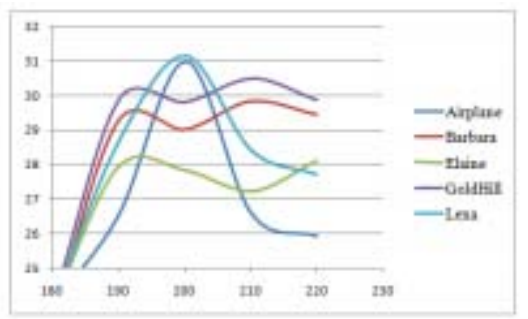

(a)

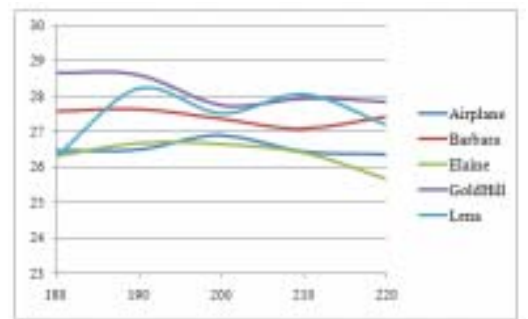

(b)

Figure 6. Cutoff frequency Vs $P S N R$ values for FFT based scheme with Lagrange interpolated PWL filter (a) for $\sigma=30$; (b) for $\sigma=50$.

FFT based scheme is better. So, we have used 200 as cutoff frequency for the Gaussian filter in all the FFT based schemes.

\section{CONCLUSION}

We have presented two image denoising techniques for noise removal. The experimental results show FFT based image denoising scheme gives higher $P S N R$ values than SWT-DWT and TDM scheme. We have also conducted experiments on different cutoff frequencies for the FFT based image denoising scheme with Lagrange interpolation based approximation of PWL filter. This approach gives better result than the PWL filter and spline approximation of PWL filter.

\section{REFERENCES}

[1] F. Liu, J. Liu, Anisotropic diffusion for image denosing based on diffusion tensors, J. Vis. Commun. Image R. 23 (2012): 516-521.

[2] C.R. Gonzalez, E. Woods, Digital Image Processing, Englewood Cliffs, NJ: Prentice Hall, 2002.

[3] M.C. Motwani, M.C. Gadiya, R.C. Motwani, F.C. Harris Jr., Survey of image denoising techniques, Proceedings of GSPx, Santa Clara, CA, 2004, pp. 27-30.

[4] R. Yang, L. Yin, M. Gabbouj, J. Astola, Y. Neuvo, Optimal weighted median filters under structural constraints, IEEE Trans. on Signal Process. 43; (1995); 591-604.

[5] R.C. Hardie, K.E. Barner, Rank conditioned rank selection filters for signal restoration, IEEE Trans. on Image Process. 3 (1994) 192-206.

[6] A.B. Hamza, P. Luque, J. Martinez, R. Roman, Removing noise and preserving details with relaxed median filters, Journal of Mathematical Imaging and Vision 11(2); (1999): 161-177.

[7] F. Russo, An image enhancement system based on noise estimation, IEEE Trans. on Instrumentation and Measurement 56 (4) (2007) 1423-1442.

[8] P. Thangavel, S. Gopinathan, Image enhancement system using wavelet transform and a non-linear piecewise filter, ICGST International Journal on Graphics, Vision and Image Processing, GVIP, 11(2); (2011): 1-7.

[9] A.K. Jain, Fundamentals of Digital Image Processing, Prentice-Hall, 1989.

[10] A. Jung, An introduction to a new data analysis tool: independent component analysis, Proceedings of Workshop GK ”Nonlinearity” - Regensburg, 2001.

[11] H. Demirel, G. Anbarjafari, Image resolution enhancement by using discrete and stationary wavelet decomposition, IEEE Trans. on Image Process. 20(5); (2011): 1458-1460.

[12] R. Chouhan, A. Mishra, P. Khanna, Fingerprint authentication by wavelet-based digital watermarking, International Journal of Electrical and Computer Engineering, 2(4); (2012): 523-528.

[13] A. Jaiswala, J. Upadhyayb, A. Somkuwarca Department, Image denoising and quality measurements by using filteringand wavelet based techniques, International Journal of Electronics and Communications, 68; (2014): 699705. 
[14] R.H. Chan, J. Ma, A multiplicative iterative algorithm for box-constrained penalized likelihood image restoration, IEEE Trans. on Image Process. 21(7); (2012): 3168-3181.

[15] W. Zeng, X. Lu, X. Tan, A local structural adaptive partial differential equation for image denoising, Multimedia Tools and Applications, 74(3); (2015): 743757.

[16] M. Lang, H. Guo, J.E. Odegard, C.S. Burrus, R.O. Well,Jr., Noise reduction using an undecimatd discrete wavelet transform, IEEE Signal Processing Letters, 3(1); (1996): 10-12.

[17] M. Clyde, G. Parmigiani, B. Vidakovic, Multiple shrinkage and subset selection in wavelets, Biometrika 85(2); (1998): 391-401.

[18] K. Dabov, A. Foi, V. Katkovnik, K. Egiazarian, Image denoising by sparse 3-D transform-domain collaborative filtering, IEEE Trans. on Image Process. 16(8); (2007): 2080-2095.

[19] Y. Yang, Z. Su, L. Sun, Medical image enhancement algorithm based on wavelet transform, IET Electronics Letters. 46(2); (2010): 120-121.

[20] J.C.Lazaro, J.L. San Emeterio, A. Ramos, J.L. Fernandez-Marraon, Influence of thresholding procedures in ultrasonic grain noise reduction using wavelets, Ultrasonics, 40; (2002): 263-267.

[21] D.L. Donoho, De-noising by soft-thresholding, IEEE Trans. on Information Theory, 41(3); (1995): 613-627.

[22] A. Khare, Uma Shanker Tiwary, Soft-thresholding for denoising of medical images - a multiresolution approach, Int. J. Wavelets Multiresolution Inf. Process. 3(4); (2005): 477-496.

[23] F. Luisier, T. Blu, M. Unser, A new SURE approach to image denoising: interscale orthonormal wavelet thresholding, IEEE Trans. on Image Process. 16(3); (2007): 593-606.

[24] K. Lu, N. He, L. Li, Nonlocal means-based denoising for medical images, Computational and Mathematical Methods in Medicine, (2012).

[25] L. Shang, D.S. Huang, Image denoising using non-negative sparse coding shrinkage algorithm, IEEE Computer Society Conference on Computer Vision and Pattern Recognition, 1; (2005): 1017-1022.

[26] A. Adler, Y. Hel-Or, M. Elad, A weighted discriminative approach for image denoising with overcomplete representations, IEEE Int. Conf. on Acoustics Speech and Signal Processing, 2010, pp. 782-785.

[27] S.D. Conte, Carl de Boor, Elementary Numerical Analysis: an algorithmic approach, Third Edition, McGrawHill International Editions, 1981.

[28] W. Jianwei, A noise removal algorithm for color image, TELKOMNIKA Indonesian Journal of Electrical Engineering, 12(1); (2014): 565-574.

[29] P. Jain, V. Tyagi, LAPB: Locally adaptive patch-based wavelet domain edge-preserving image denoising, Information Sciences, 294; (2015): 164181.

\section{BIOGRAPHIES OF AUTHORS}
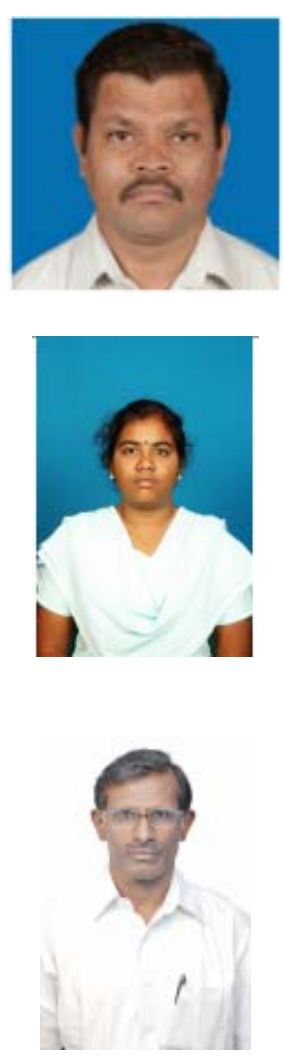

S. Gopinathan received his M.Sc and Ph.D. from Bharathiar University and University of Madras respectively. Area of scientific interest: digital image processing.

R. Kokila received her M.Sc and M.Phil from University of Madras during 2005 and 2006 respectively. She is pursing Ph.D at University of Madras. Area of scientific interest: digital image processing and artificial neural networks.

P. Thangavel received his M.Tech and Ph.D. from Indian Institute of Technology and Bharathidasan University respectively. He is currently working as Professor and Head of the Department of Computer Science, University of Madras. Area of scientific interest: algorithms and artificial systems. 